\title{
Scattering and attenuation of surface acoustic waves in droplet actuation
}

\author{
Z. J. Jiao, X. Y. Huang and N. -T. Nguyen \\ $\dagger$ School of Mechanical and Aerospace Engineering, Nanyang Technological \\ University, 50 Nanyang Avenue, Singapore 639798 \\ E-mail: MXHUANG@ntu.edu.sg
}

\begin{abstract}
.
This paper presents an analytical model for surface acoustic waves (SAW) in actuating a single liquid droplet on a piezoelectric substrate. Both scattering waves outside the droplet and attenuation waves beneath the droplet are obtained, and the energy transfer from SAW to droplet in the process of actuation is calculated. The results from this analytical model can provide qualitative explanations to some experimental observations, such as the weak actuation behind the droplet and flow patterns inside the droplet. It is found that effective actuation wavelength is around $1 / 5$ of the droplet radius, at which the droplet absorbs the maximum incident SAW energy.
\end{abstract}

PACS: 43.35.Pt

keywords: surface acoustic wave; microfluidics 


\section{Introduction}

Micro-scale tools has a growing importance for life science research in the last decade. Droplet-based microfluidics has been emerged as an alternative to continuous-flow microfluidics for bio-chemical analysis. A microdroplet is a good option for the carrier of reagents in micro-scale. A microdroplet also provides a platform for chemical reactions. The emerging field of droplet-based microfluidics leads to the need of effective actuation and manipulations of individual droplets in micro-scale [1]. Active control of microdroplets can be achieved by using effects such as electrowetting and thermocapillarity. Electrowetting utilizes electrostatic forces at the solid/liquid/liquid or solid/gas/liquid interfaces to manipulate the interfacial energy and subsequently the motion of droplets [2]. In the thermocapillary actuation, gradients of surface tension induced by temperature gradients can move droplets on a flat surface [3]. Our group has reported an one-dimensional (1D) analytical model for the transient behaviour of a liquid plug in a capillary which is heated at one end [4]. However, these methods still have some drawbacks. For instance, high voltage and dielectric liquid are required for electrowetting actuation, while high temperature may be needed for thermocapillary actuation. The high temperature may cause damages to biological samples. These problems could be avoided by using surface acoustic wave(SAW)to actuate and manipulate the droplets on a planar surface.

Apart from applications in electronic communication devices for signal processing, SAW power propagating on a piezoelectric substrate can also actuate droplets on the surface. The SAW has a longitudinal wave component, whose displacement is parallel to the propagation vector, and a transverse wave component, with displacement perpendicular to the propagation vector. When fluid is introduced onto the surface, the longitudinal component of the surface acoustic wave is coupled to the fluid by the viscosity. The transverse component is attenuated by acoustic impedance mechanism. This propagation mode is called a leaky Rayleigh wave [5]. Since the SAW speed $c_{\mathrm{s}}$ in a solid substrate is larger than the sound speed in a liquid $c_{1}$, diffraction longitude wave always radiates into the liquid with an angle of $\Phi=\arcsin \left(c_{\mathrm{l}} / c_{\mathrm{s}}\right)$. The SAW induced acoustic streaming has been used to pump liquid in microchannels by Nguyen et al. [6, 7]. In their work, the effects of the channel height, wave amplitude and back pressure on the velocity profile and flow rate were investigated. The SAW has also been used to propel liquid droplets on a planar surface. Wixforth conducted several experiments on this phenomena $[8,9]$. In his experiments, a $128^{\circ}$ rotated Y-cut X-propagating $\mathrm{LiNbO}_{3}$ two-inch wafer was used as the substrate. Both water and glycerol were used as droplet liquid. It was demonstrated that a relatively large droplet velocity could be achieved by using SAW actuation with a relatively low power input. Furthermore, liquid droplets with different viscosities could be actuated to travel with different velocities. Besides the actuation, efficient mixing can be achieved by using SAW [10].

A careful review of the literature available on SAW actuation of liquid droplets reveals that most of the results reported were based on experimental observations and, to 
the best knowledge of the authors, analytical models related to SAW scattering and attenuation in droplet actuation are yet to be reported. In this paper, we present an analytical model for acoustic velocity potential fields associated with SAW inside and outside of the droplet when it is actuated by an incident SAW. The paper attempts to understand and to explain those effects observed in the experiments by examining the SAW fields and energy losses during the process of actuation.

\section{Analytical modeling}

\subsection{Attenuation of $S A W$ at a liquid-solid interface}

Figure 1 shows the schematics concept of the actuation where an incident SAW is propagating to a droplet. Part of the incident SAW are scattered by the air-liquidsolid contact line, while the rest are penetrated into the area covered by the droplet. The penetrated SAW decays under the droplet because of the viscous loading. The attenuation is induced to both longitude and transverse wave components. The damping coefficients for the longitude wave $\alpha_{1}$ and the transverse wave $\alpha_{\mathrm{t}}$ are respectively [11]:

$$
\begin{aligned}
\alpha_{\mathrm{t}} & =\frac{\rho_{\mathrm{f}} c_{\mathrm{f}}}{\rho_{\mathrm{s}} v_{\mathrm{s}}} \frac{1}{\lambda} \quad(1 / \mathrm{mm}), \\
\alpha_{\mathrm{l}} & =\frac{\sqrt{\rho_{\mathrm{f}} \eta_{\mathrm{f}} \omega^{3}}}{4 \sqrt{2} \pi^{2} \rho_{\mathrm{s}} c_{\mathrm{s}}^{2}} \quad(1 / \mathrm{mm}) .
\end{aligned}
$$

Where, $\rho_{\mathrm{s}}, \lambda, v_{s}, \rho_{\mathrm{f}}, c_{\mathrm{f}}, \omega=2 \pi f$ and $\eta_{\mathrm{f}}$ are the density of substrate, the SAW wavelength, the SAW velocity, the density of liquid, the sound velocity in the liquid, the angular velocity and the dynamic viscosity of the liquid, respectively. Based on a water droplet, both transverse and longitude damping factors can be estimated as $\alpha_{\mathrm{t}}=0.8061 / \mathrm{mm}$, $\alpha_{1}=9.60 \times 10^{-4} 1 / \mathrm{mm}$ for actuation at a frequency of $f=40 \mathrm{MHz}$. Since $\alpha_{1} \ll \alpha_{\mathrm{t}}$, the attenuation of the longitudinal waves penetrated into the liquid droplet are much smaller than the transverse components, the SAW diffracted into the droplet are therefore dominated by transverse waves. In other words, the actuation power is mainly contributed by the transverse waves, and longitudinal wave has little contribution to the actuation. So that in the following model, only the transverse waves are considered, while the longitudinal waves are neglected [11].

\subsection{Scattering and internal waves}

As shown in Figure 1, we consider a two-dimensional wave scattering problem. The velocity potentials associated with the incident wave, the scattering wave and the penetrated wave are denoted by $\psi_{\text {incident }}, \psi_{\text {scattering }}$ and $\psi_{\text {internal }}$ respectively. The droplet is of a radius $a$ and the wavelength of the incident SAW is $\lambda$. For the convenience of the 
calculation, time factor $e^{-i \omega t}$ will be dropped hereafter and all three waves are expressed in a cylindrical coordinate system as

$$
\begin{aligned}
& \psi_{\text {incident }}=\psi_{0} e^{i \mathrm{k} \cdot \mathrm{x}}=\psi_{0} e^{i k r \cos \theta}=\psi_{0} \sum_{m=-\infty}^{\infty} i^{m} J_{m}\left(k_{\mathrm{o}} r\right) e^{i m \theta} \\
& \psi_{\text {scattering }}=\sum_{m=-\infty}^{\infty} A_{m} i^{m} H_{m}\left(k_{\mathrm{o}} r\right) e^{i m \theta} \\
& \psi_{\text {internal }}=\sum_{m=-\infty}^{\infty} B_{m} i^{m} J_{m}\left(k_{\mathrm{i}} r\right) e^{i m \theta}
\end{aligned}
$$

Here, $J_{m}\left(k_{\mathrm{o}} r\right)$ is the first kind of Bessel function of order $m, \psi_{0}$ is the amplitude of the incident wave velocity potential. $k_{\mathrm{o}}=\frac{2 \pi}{\lambda}$ and $k_{\mathrm{i}}=\frac{2 \pi}{\lambda}+i \alpha_{t}$ are the wave numbers outside and inside the area covered by the droplet respectively. $H_{m}\left(k_{\mathrm{o}} r\right)$ is the first kind of Hankle function of order $m . A_{m}$ and $B_{m}$ are two unknown coefficients which will be determined based on the continuity conditions of velocity and pressure at the edge of the droplet base. The total acoustic wave field outside the droplet is denoted as $\psi_{\text {external }}$, which is the sum of $\psi_{\text {incident }}$ and $\psi_{\text {scattering }}$, i.e.,

$$
\psi_{\text {external }}=\psi_{0} \sum_{m=-\infty}^{\infty} i^{m} J_{m}\left(k_{\mathrm{o}} r\right) e^{i m \theta}+\sum_{m=-\infty}^{\infty} A_{m} i^{m} H_{m}\left(k_{\mathrm{o}} r\right) e^{i m \theta}
$$

By calculating the radial velocity $\left.u_{r}\right|_{r=a}=-\left.\frac{\partial \psi}{\partial r}\right|_{r=a}$ and pressure $p=-\left.i \rho \omega \psi\right|_{r=a}$, the continuity conditions are written as:

$$
\begin{aligned}
& \frac{\partial}{\partial r}\left[\psi_{0} \sum_{m=-\infty}^{\infty} i^{m} J_{m}\left(k_{\mathrm{o}} r\right) e^{i m \theta}+\sum_{m=-\infty}^{\infty} A_{m} i^{m} H_{m}\left(k_{\mathrm{o}} r\right) e^{i m \theta}\right]_{r=a}= \\
& \frac{\partial}{\partial r}\left[\sum_{m=-\infty}^{\infty} B_{m} i^{m} J_{m}\left(k_{\mathrm{i}} r\right) e^{i m \theta}\right]_{r=a}
\end{aligned}
$$

and

$$
\begin{aligned}
& {\left[\psi_{0} \sum_{m=-\infty}^{\infty} i^{m} J_{m}\left(k_{\mathrm{o}} r\right) e^{i m \theta}+\sum_{m=-\infty}^{\infty} A_{m} i^{m} H_{m}\left(k_{\mathrm{o}} r\right) e^{i m \theta}\right]_{r=a}=} \\
& {\left[\sum_{m=-\infty}^{\infty} B_{m} i^{m} J_{m}\left(k_{\mathrm{i}} r\right) e^{i m \theta}\right]_{r=a}}
\end{aligned}
$$

The two continuity conditions lead to the following two simultaneous equations to solve $A_{m}$ and $B_{m}$.

$$
\left\{\begin{array}{l}
-k_{\mathrm{o}} A_{m} H_{m}^{\prime}\left(k_{\mathrm{o}} a\right)+k_{\mathrm{i}} B_{m} J_{m}^{\prime}\left(k_{\mathrm{i}} a\right)=\psi_{0} k_{\mathrm{o}} J_{m}^{\prime}\left(k_{\mathrm{o}} a\right) \\
A_{m} H_{m}\left(k_{\mathrm{o}} a\right)-B_{m} J_{m}\left(k_{\mathrm{i}} a\right)=-\psi_{0} J_{m}\left(k_{\mathrm{o}} a\right)
\end{array}\right.
$$


The final results are:

$$
\left\{\begin{array}{l}
\psi_{\text {external }}=\psi_{0} \sum_{m=-\infty}^{\infty} i^{m} J_{m}\left(k_{\mathrm{o}} r\right) e^{i m \theta}+\sum_{m=-\infty}^{\infty} A_{m} i^{m} H_{m}\left(k_{\mathrm{o}} r\right) e^{i m \theta} \\
\psi_{\text {internal }}=\sum_{m=-\infty}^{\infty} B_{m} i^{m} J_{m}\left(k_{\mathrm{i}} r\right) e^{i m \theta}
\end{array}\right.
$$

where $\psi_{\text {external }}=\psi_{\text {incident }}+\psi_{\text {scattering }}$, and

$$
\left\{\begin{aligned}
A_{m} & =\frac{\psi_{0}\left[k_{\mathrm{o}} J_{m}\left(k_{\mathrm{i}} a\right) J_{m}^{\prime}\left(k_{\mathrm{o}} a\right)-k_{\mathrm{i}} J_{m}^{\prime}\left(k_{\mathrm{i}} a\right) J_{m}\left(k_{\mathrm{o}} a\right)\right]}{k_{\mathrm{i}} J_{m}^{\prime}\left(k_{\mathrm{i}} a\right) H_{m}\left(k_{\mathrm{o}} a\right)-k_{\mathrm{o}} J_{m}\left(k_{\mathrm{i}} a\right) H_{m}^{\prime}\left(k_{\mathrm{o}} a\right)} \\
B_{m} & =\frac{\psi_{0} k_{\mathrm{o}}\left[H_{m}\left(k_{\mathrm{o}} a\right) J_{m}^{\prime}\left(k_{\mathrm{o}} a\right)-H_{m}^{\prime}\left(k_{\mathrm{o}} a\right) J_{m}\left(k_{\mathrm{o}} a\right)\right]}{k_{\mathrm{i}} H_{m}\left(k_{\mathrm{o}} a\right) J_{m}^{\prime}\left(k_{\mathrm{i}} a\right)-k_{\mathrm{o}} H_{m}^{\prime}\left(k_{\mathrm{o}} a\right) J_{m}\left(k_{\mathrm{i}} a\right)}
\end{aligned}\right.
$$

\subsection{Liquid static pressure field at solid-liquid interface}

A previous experimental study demonstrated that the SAW not only sets the droplet in motion but also induces a flow field inside the droplet [10]. The understanding of the SAW-induced flow patterns within the droplet requires further study on the interaction between SAW and liquid, probably involving in acoustic streaming or other nonlinear mechanism. In the present study, we attempt to look into the possible effect of SAW on the droplet liquid by examining the static pressure induced by the internal SAW at the solid-liquid interface, where the SAW generates the same pressure and velocity in the liquid. The static pressure can be written as [13],

$$
P_{s}=\frac{\left\langle p^{2}\right\rangle}{2 \rho_{\mathrm{f}} c_{\mathrm{f}}^{2}}-\frac{1}{2} \rho_{\mathrm{f}}\left\langle v^{2}\right\rangle
$$

where \langle\rangle denotes time average over one period of acoustic oscillation, $p$ is the sound pressure and $v$ is the medium particle velocity. $\rho_{\mathrm{f}}$ and $c_{\mathrm{f}}$ are average medium density and sound speed in liquid, respectively. The static pressure can be approximated by dropping the medium velocity term which is normally very small in SAW, so that

$$
P_{\mathrm{s}} \approx \frac{\langle p\rangle^{2}}{2 \rho_{\mathrm{f}} c_{\mathrm{f}}^{2}} \propto\left\langle\psi_{\text {internal }}\right\rangle^{2} .
$$

\subsection{Results and discussion}

The results from the above model are plotted and discussed in this section. Figure 2 shows the simulation of the total surface acoustic velocity potential field for a typical case $a / \lambda=5$. The external waves are shown in Figure 2(a) and the detailed internal acoustic field is plotted in Figure 2(b). It is seen from the total field that an acoustic shadow region is formed right after the droplet along the direction of incident wave propagation. The SAW strength in the shadow region is observed by plotting $\left|\psi_{\text {external }}\right|^{2} /\left|\psi_{\text {incident }}\right|^{2}$ along $\theta=0$, which is shown in Figure 3. It is seen that, for $a / \lambda \geq 5$, SAW behind the 
droplet becomes much weaker than the incident wave, due to the scattering and absorption by the droplet. This may explain the experimental observation that the actuation would not be effective to the second droplet behind the first one [8], illustrating in Figure 4(a). The liquid streaming patterns observed by Wixforth are also sketched in Figure 4(b) to compare our results from the internal SAW. The static pressures beneath the droplet are calculated using (13) and plotted in Figure 5 for three different wavelengths. It shows that, for $a / \lambda \geq 5$, a relatively high pressure zone is formed along the centre line of droplet in the propagation direction and the pressure decays from the left side after SAW enters the droplet. The pressure gradient along the centre line may induce the liquid flow observed from the top view illustrated in Figure $4(\mathrm{~b})$. For $a / \lambda=5$, Figure 5 depicts two high-pressure spots symmetrically around the centre line. The high pressure spots may drive the liquid to circulate from the bottom to top, as illustrated in the front view of Figure 4(b). It is seen from Figure 5 that the static pressures are either uniform across the droplet at a longer wavelength $(a / \lambda=2)$, or decay too fast at a shorter wavelength $(a / \lambda=10)$. The penetrated SAW may not generate flow patterns in the droplet in these cases.

\section{Energy absorbed by droplet}

The interaction between the incident SAW and droplet can be better understood by examining the energy absorbed by droplet in the process of actuation. This is calculated by integrating the power flow across the droplet boundary, which can be written as [15],

$$
P_{\text {absorbed }}=-\frac{a}{2} \operatorname{Re}\left\{\int_{0}^{2 \pi}\left[p \cdot(u)^{*}\right]_{r=a} d \theta\right\} .
$$

The superscript $*$ denotes the complex conjugate and the minus sign indicates the power flowing into the droplet. The integration boundary is a circle with a radius $r=a$. The radial velocity and the pressure are computed from the internal SAW $\psi_{\text {internal }}$ obtained in the previous section. So that

$$
\begin{aligned}
& P_{\text {absorbed }}=-\frac{a}{2} \operatorname{Re}\left\{\int_{0}^{2 \pi}\left[p \cdot(u)^{*}\right]_{r=a} d \theta\right\} \\
& =-\frac{a}{2} \operatorname{Re}\left\{\int_{0}^{2 \pi}\left[i \rho \omega k_{\mathrm{i}}^{*} \sum_{m=-\infty}^{\infty} \sum_{n=-\infty}^{\infty} B_{m} B_{n}^{*} i^{m-n} J_{m}\left(k_{\mathrm{i}} a\right)\left[J_{n}^{\prime}\left(k_{\mathrm{i}} a\right)\right]^{*} e^{i(m-n) \theta}\right] d \theta\right\} \\
& =-\frac{a \rho \omega}{2} \operatorname{Re}\left\{\int_{0}^{2 \pi}\left[i k_{\mathrm{i}}^{*} \sum_{m=-\infty}^{\infty} B_{m} B_{m}^{*} J_{m}\left(k_{\mathrm{i}} a\right)\left[J_{m}^{\prime}\left(k_{\mathrm{i}} a\right)\right]^{*}\right] d \theta\right\} \\
& =-\pi a \rho \omega \operatorname{Re}\left\{i k_{\mathrm{i}}^{*} \sum_{m=-\infty}^{\infty} B_{m} B_{m}^{*} J_{m}\left(k_{\mathrm{i}} a\right)\left[J_{m}^{\prime}\left(k_{\mathrm{i}} a\right)\right]^{*}\right\} .
\end{aligned}
$$


The incident power hitting on the droplet with diameter $2 a$ is obtained from $\psi_{\text {incident }}$ as

$$
\begin{aligned}
& P_{\text {incident }}=2 a \frac{\rho}{2}\left(v^{2}+\frac{1}{\rho^{2} c^{2}} p^{2}\right) \\
& =a \rho c\left(k_{\mathrm{o}}^{2} \psi_{0}^{2}+\frac{\omega^{2}}{c^{2}} \psi_{0}^{2}\right)=2 a \rho c k_{\mathrm{o}}^{2} \psi_{0}^{2} .
\end{aligned}
$$

The fractional ratio of the droplet absorbed power $P_{\text {absorbed }}$ to the incident power $P_{\text {incident }}$ is denoted as $\gamma$, and is obtained as

$$
\begin{aligned}
& \gamma=\frac{P_{\text {absorbed }}}{P_{\text {incident }}}=-\frac{\pi a \rho \omega \operatorname{Re}\left\{i k_{\mathrm{i}}^{*} \sum_{m=-\infty}^{\infty} B_{m} B_{m}^{*} J_{m}\left(k_{\mathrm{i}} a\right)\left[J_{m}^{\prime}\left(k_{\mathrm{i}} a\right)\right]^{*}\right\}}{\psi_{0}^{2} a \rho c k_{\mathrm{o}}^{2}} \\
& =-\frac{\pi \operatorname{Re}\left\{i k_{\mathrm{i}}^{*} \sum_{m=-\infty}^{\infty} B_{m} B_{m}^{*} J_{m}\left(k_{\mathrm{i}} a\right)\left[J_{m}^{\prime}\left(k_{\mathrm{i}} a\right)\right]^{*}\right\}}{\psi_{0}^{2} k_{\mathrm{o}}} .
\end{aligned}
$$

The power absorbed by the droplet is viewed by plotting $\gamma$ versus $a / \lambda$ with different values of attenuation factor $\alpha_{\mathrm{t}}$ and the results are shown in Figure 6 . It is seen that the power absorptions increase with $a / \lambda$ and reach to maximum values around $a / \lambda=5$. The power absorptions decrease afterwards when the wavelength becomes shorter and most of the incident waves are scattered away. The results presented in Figure 6 also show that the maximum power absorptions can be from $70 \%$ to $90 \%$ for the attenuation factor taking values $\alpha_{\mathrm{t}}=0.81 / \mathrm{mm}$ (for a water droplet) or greater. These results are qualitatively comparable to the reported experimental data [9], in which $10 \%$ of the incident energy hitting on the droplet was found scattered away by a water droplet under SAW actuation at $a / \lambda=5$.

\section{Conclusions}

An analytical study has been presented in this paper to simulate acoustic surface waves (SAW) in the process of actuating a liquid droplet on a piezoelectric substrate. Both scattering waves outside the droplet and attenuation waves beneath the droplet are calculated. The results are compared to the experimental observations. Qualitative agreements are obtained in terms of the acoustic shadow behind the droplet and possible connection between the flow patterns and static pressures generated beneath the droplet. The study on the energy absorbed by the droplet shows that the maximum energy absorption occurs at the droplet radius-to-wavelength ratio of $a / \lambda=5$. This agrees with the experimental results, and also indicates that the droplet actuation by SAW may in general be effective at $a / \lambda=5$. Around this wavelength the absorbed energy will be strong enough to actuate the droplet and induce an internal flow. The results generated in this study may be useful in further investigations on droplet actuation by SAW. 


\section{Acknowledgment}

The authors would like to thank the Ph.D scholarship of School of Mechanical and Aerospace Engineering, Nanyang Technological University, Singapore.

\section{Reference}

[1] Song H, Chen DL, and Ismagilov RF, 2006 Reactions in droplets in microfluidic channels Angew. Chem. Int. Ed. 45:7336-7356.

[2] Pollack MG, Fair RB and Shenderov AD 2000 Electrowetting-based actuation of liquid droplets for microfluidic applications Applied Physics Letter 77:1725-1727

[3] Darhuber AA, Valentino JP, Davis JM and Troian SM 2003 Microfluidic actuation by modulation of surface stresses Applied Physics Letter 82:657-659

[4] Nguyen NT and Huang XY 2005 Thermocapillary effect of a liquid plug in transient temperature field Japanese Journal of Applied Physics 44:1139-1142

[5] Cheeke JDN and Morisseau P 1982 Attenuation of Rayleigh waves on a LiNbO3 crystal in contact with a liquid He bath Journal of Low Temperature Physics 46:3-4

[6] Nguyen NT, Meng A, Black J, White RM 1999 Integrated thermal flow sensor for in-situ measurement and control of acoustic streaming in flexural-plate-wave pumps Sensors and Actuators A: Physical 79:115-121

[7] Nguyen NT, White, RM 2000 Acoustic streaming in micromachined flexural plate wave devices: numerical simulation and experimental verification IEEE Transactions on Ultrasonics, Ferroelectrics and Frequency Control 47:1463-1471

[8] Strobl CJ, Rathgeber A, Wixforth A, Gauer C and Scriba J 2002 Planar microfluidic processors IEEE Ultrasonics Symposium 255.

[9] Wixforth A 2003 Acoustically driven planar microfluidics Superlattices and Microstructures 33 $(5-6): 389-396$.

[10] Frommelt F, Kostur M, Wenzel-Scha M, Talkner P, Hanggi P, and Wixforth A 2008 Microfluidic mixing via acoustically driven chaotic advection Physical Review letter 100:034502

[11] Arzt RM, Salzmann E, and Dransfeld K 1967 Elastic surface waves in quartz at $316 \mathrm{MHz} A p p l$. Phys. Lett. 10(5):165

[12] Wixforth A 2006 Acoustically driven programmable microfluidics for biological and chemical applications Journal of the Association for Laboratory Automation 11:6

[13] Xie WJ, Cao CD, L YJ, Hong ZY, and Wei B 2006 Acoustic method for levitation of small living animals Appl. Phys. Lett. 89: 214102

[14] Browers NG, Himberger DE, and Mayer WG 1979 IEEE Trans. Sonics Ultrasonics SU-26(4):306

[15] Huang XY 1992 Energy dissipation in sound scattering by a submerged cylindrical shell ACUSTICA 77:221

[16] Brochardc F 1989 Motions of droplets on solid surfaces induced by chemical or thermal gradients Langmuir 5: 433

[17] Beyssen D, Brizoual LL, Elmazria O and Alnot P 2006 Microfluidic device based on surface acoustic wave Sensors and Actuators B $118: 380-385$ 


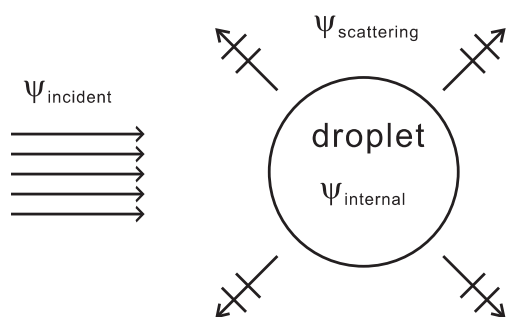

(a)

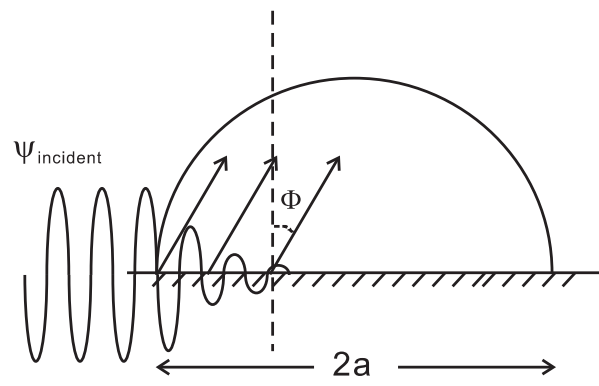

(b)

Figure 1. Schematic illustration of a droplet actuated by surface acoustic waves: (a) top view and (b) side view.

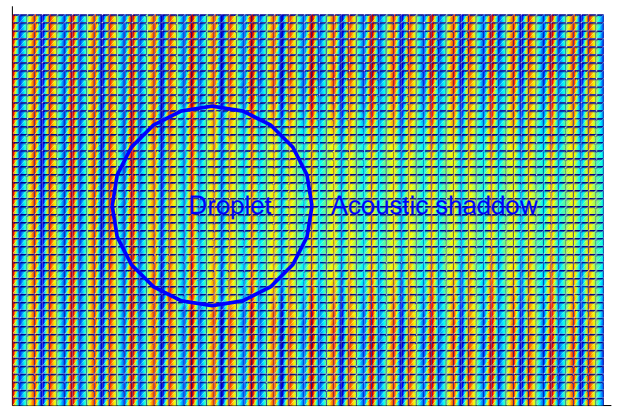

(a)

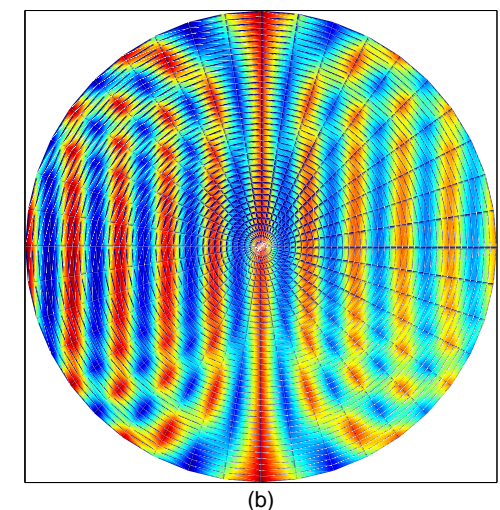

Figure 2. Simulation results of the wave field: (a) Total acoustic velocity potential field; (b) Acoustic velocity potential beneath the droplet $(a / \lambda=5$ and attenuation factor $\alpha_{\mathrm{t}}=0.8061 / \mathrm{mm}$ ). 


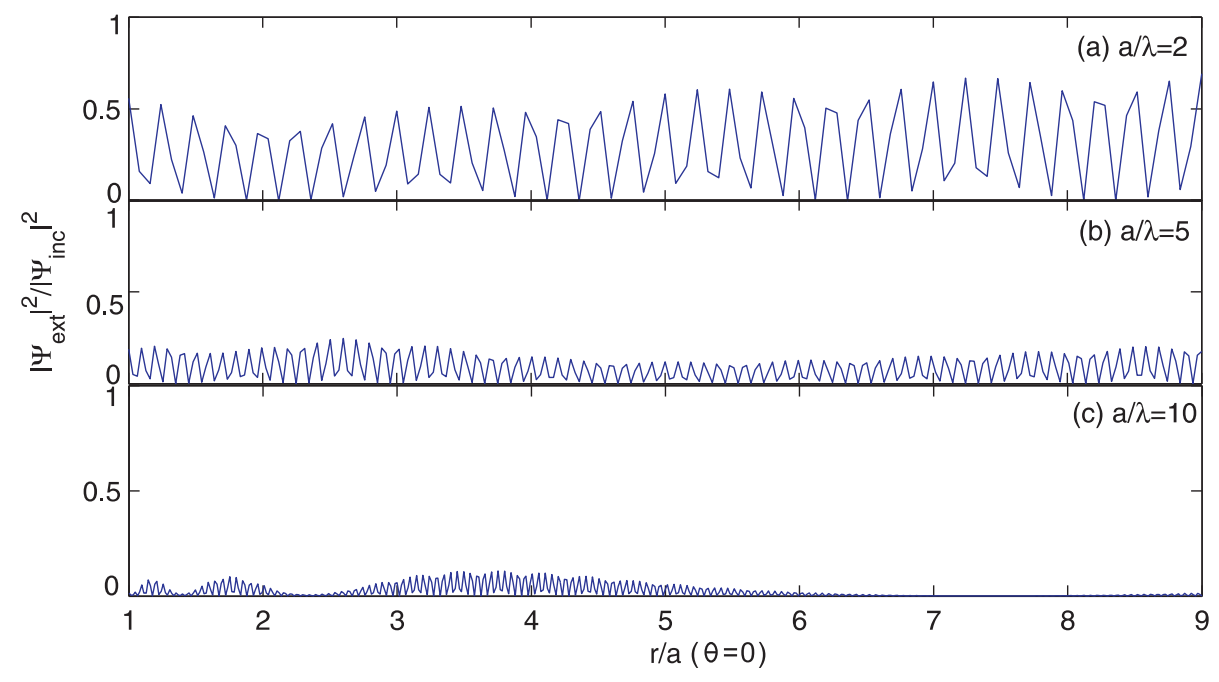

Figure 3. Variation of SAW strength with $r / a$ behind the droplet.

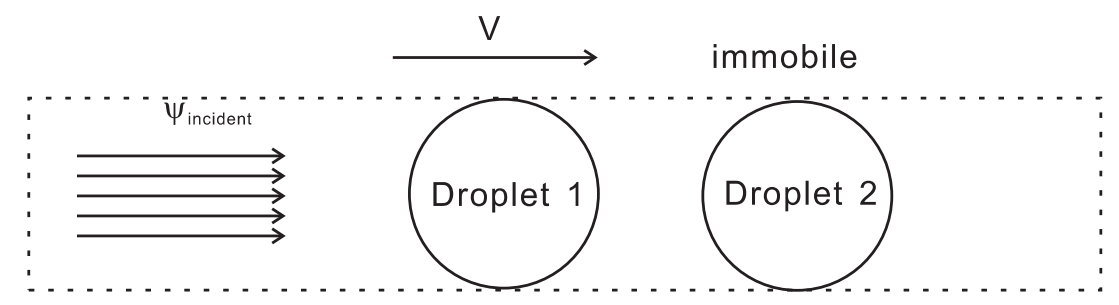

(a)

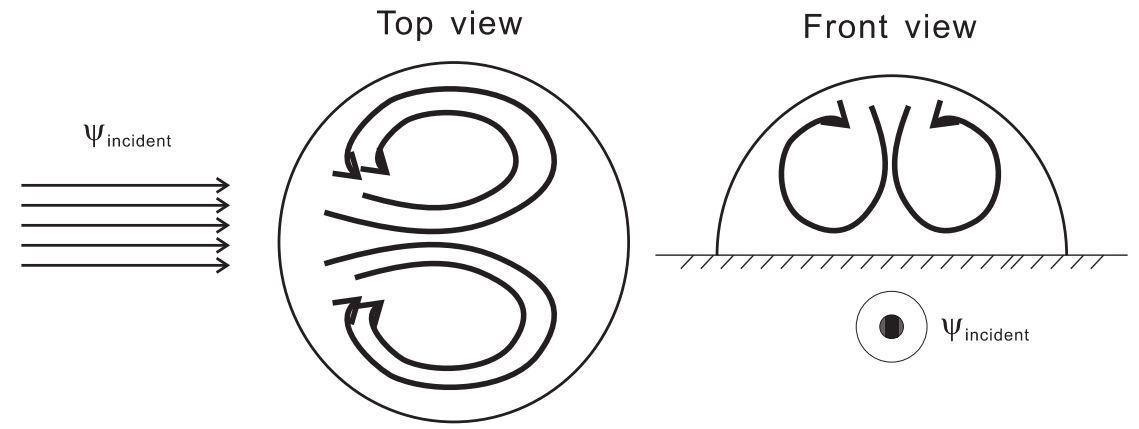

(b)

Figure 4. Sketch of the experimental results obtained by Wixforth [8, 9]. Droplet radius $a=0.5 \mathrm{~mm}$ and SAW wavelength $\lambda=0.1 \mathrm{~mm}$. (a) The second droplet lies in the acoustic shadow of the first droplet. (b) SAW induced internal streaming flow patterns in both top view and front view. 


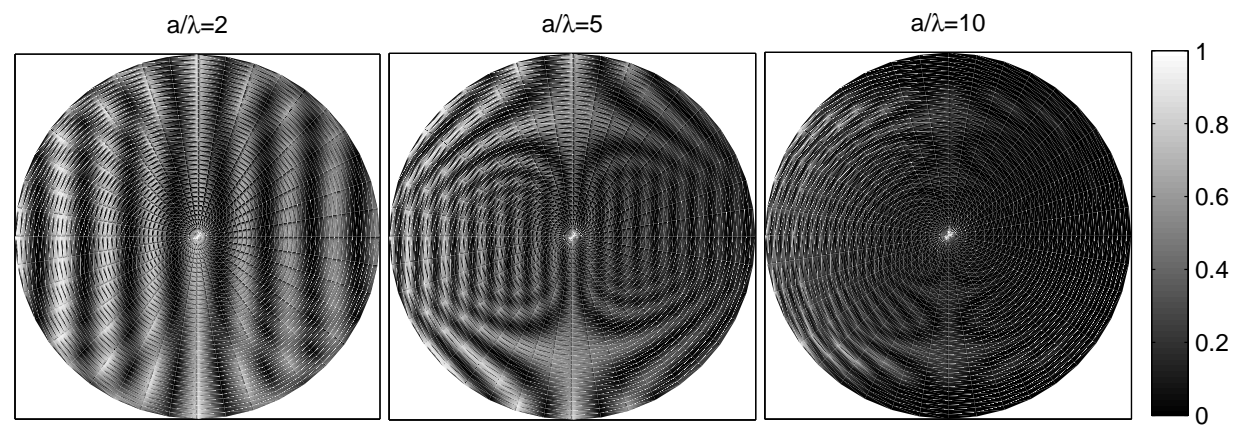

Figure 5. Plots of $\left\langle\psi_{\text {internal }}\right\rangle^{2} / \psi_{0}^{2}$ showing static pressures beneath the droplet for three different actuation wavelengths.

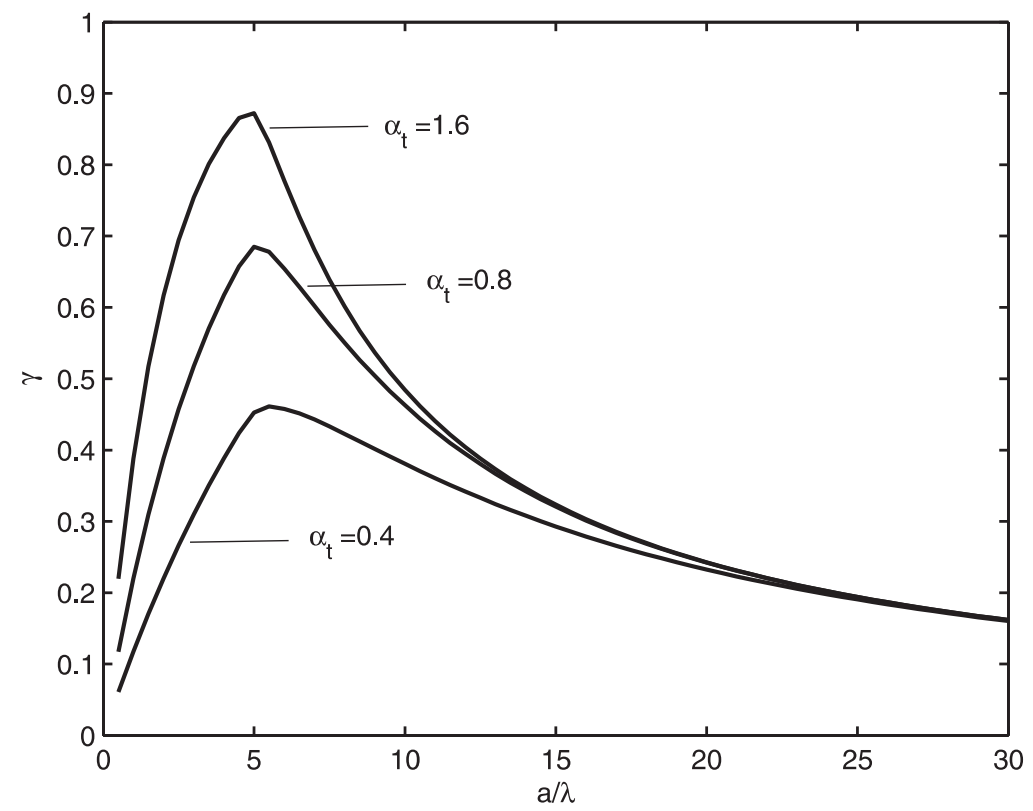

Figure 6. Ratio of the absorbed power to the incident power versus a/ $\lambda$. 